\title{
Risk Factors of Delayed Language Development among Preschool Children Attending Assiut University Hospitals
}

\author{
HALA H. ABOUFADDAN, M.D. and SABRA M. AHMED, M.D. \\ The Department of Public Health \& Community Medicine, Faculty of Medicine, Assiut University, Assiut, Egypt
}

\begin{abstract}
Background: To study the risk factors associated with Delayed Language Development (DLD) among children attending Outpatient Phoniatric Clinic of Assiut University hospitals.

Subjects and Methods: A case-control study was conducted on 150 cases with DLD aged 3-5 years attended Outpatient Phoniatric Clinic and 150 control children of the same age range with no DLD attended outpatient clinic of AssiuT University Children's Hospital. A structured interview questionnaire was used for collection of relevant data from children's caregivers.
\end{abstract}

Results: This study showed that urban residence, low birth weight, male gender, delivery by Cesarean section, parent consanguinity and presence cyanosis after birth were significantly associated with DLD.

Conclusion: It was concluded that male gender, history of perinatal events, and parent consanguinity are significant risk factors for DLD. Awareness about risk factors of DLD need to be raised among parents and health care providers for prevention, early detection and proper management.

Key Words: Delayed language development - Risk factors Preschool children.

\section{Introduction}

LANGUAGE is defined as the conceptual processing on communication. It consists of receptive language (understanding) and expressive language (the capacity to convey information, ideas, feelings and thoughts). On the other hand, speech is a method of verbal language communication [1].

Language and speech delays as well as disorders are common, including an estimated prevalence

Correspondence to: Dr. Hala H. Aboufaddan, The Department of Public Health \& Community Medicine, Faculty of Medicine, Assiut University, Assiut, Egypt within $5 \%$ to $12 \%$ (median, $6 \%$ ) in children aged 2-5 years [2]. Delayed speech and language development are considered as the most common disabilities of children. Prevention of delayed speech and language disorder is considered a public health problem because of their high prevalence and economic cost [3]

Delayed speech and language children are those whosoever develops speech or language at a slower rate than expected however in the correct sequence, on the other hand speech disorders are defined as development of speech or language in a manner that is qualitatively beyond typical development [4].

Risk of learning disabilities is increased among speech and language delayed children once they reach school age [5].

Early detection of children at risk for speech and language impairments is important to provide early interventions essential to limit the negative impacts of delayed speech development [6]. Early examination of children at risk can lead to detection of large proportion children with delayed speech by age of 24-30 months [7].

Several factors affect language development, including birth order, premature delivery, birth weight, parental education, gender of the child, environmental factors, and family history of delayed language development [8]

This study focus on preschool children (3-5 years) which will allow for identification of those children at risk of having delayed speech to identify those in need of early intervention. A better understanding of the risk factors associated with delayed 
speech will help in the development of effective prevention program.

General objective: To promote proper health status among preschool children including proper speech and language development.

Specific objectives: To study the sociodemographic, obstetric and medical risk factors associated with delayed language development among preschool children (3-5 years) attending Outpatient Phoniatric Clinic of Assiut University Hospitals.

\section{Subjects and Methods}

Children attending the Phoniatric Outpatient Clinic of Assiut University Hospitals during the morning shift, during a four-month period, fulfilling the inclusion criteria-age range of 3-5 years and seeking medical advice for delayed language development was included in this study. The number of cases was 150. A control group of 150 children of the same age group (3-5 years) with normal language development (confirmed by physical examination) was taken from attendants of Outpatient Clinics of Assiut University Children's Hospital.

\section{Exclusion criteria:}

- Children outside the age range.

- Children previously diagnosed with neurodevelopmental problems for example cerebral palsy and children with cognitive delays as autism.

Research design: A case-control study design was used.

Study setting: The study was conducted at the Outpatient Phoniatric Clinic of Assiut University Hospital and Outpatient Clinic of Assiut University Children's Hospital. The duration of data collection was 4 months, from February to May 2016.

Sample size: Sample size was calculated using EPI INFO version 3.5.1 (2008) for unmatched case control study. Sample size calculation was based on prevalence of risk factor among control children (for example male gender among children aged 35 years of $52 \%$ and odds ratio of 2.19) based on previous similar study [9]. With a power of $80 \%$ and confidence level of $95 \%$, the sample size was found to be 120 for cases and 120 for controls. The sample was expanded to 150 for cases and 150 for controls.
Sampling technique: Total coverage of all cases attending the Phoniatric Outpatient Clinic of Assiut University Hospitals aged (3-5 years) of both sexes and diagnosed as DLD by phoniatrician during study period.

Control group of children of the same age group (3-5 years) of both sexes with normal language development (confirmed by physical examination of phoniatrician) was taken from attendants of Outpatient Clinics of Assiut University Children's Hospital.

Tool of the study: Data were collected from caregivers of children by using structured interview questionnaire. It covered the following items:

- Socio-demographic characteristics of the child's family: Residence, education and occupation of both parents, family type ...... etc.

- Risk factors: Whether prenatal as maternal age at birth, medical and obstetric history of mother during child pregnancy. Natal factors as delivery mode, birth attendance, place of delivery, birth trauma, birth weight, gestational age, hypoxia and cyanosis. Post-natal factors as jaundice, type of feeding after birth.

- The child's medical history: Age, gender, history of admission into incubator or hospital, history of meningitis and convulsions.

Socio-economic level of the families of the studied children was determined by using the socioeconomic status scale developed by El-Gilany et al. [10].

Pilot study: A pilot study was conducted on 20 children to check for difficulties in the questionnaire that may arise during final data collection. Necessary modifications were applied before final data collection. Those children were not included in the final study.

Ethical consideration: Approval of the Ethical Review Committee of Faculty of Medicine, Assiut University was obtained. The necessary official permissions from different authorities were obtained before the conduction of the study. Explanation of the aim of the study to child's caregiver was performed. Informed consent to participate in the study was obtained from caregivers of children. Confidentiality of the data was assured.

Statistical analysis: Data were verified, coded, and entered by using SPSS program Version 20. 
Chi square test was used for comparison between categorical variables and $t$-test for comparison between quantitative variables. Multi-variate logistic regression analysis was used for significant variables. $p$-value less than 0.05 was considered significant.

\section{Results}

Table (1) shows sociodemographic characteristics of studied children. Male gender was significantly higher among cases than control group (70\% versus $51.3 \%, p<0.001)$. Cases had significantly higher percentage of urban residence than controls $(26.7 \%$ versus $8 \%)(p<0.001)$. First birth orders among cases were significantly higher than controls $(61.3 \%$ versus $38.7 \%)(p<0.001)$. Employed fathers and mothers were significantly higher among cases than controls $(57.3 \%$ versus $47.3 \%, p=0.021$ for fathers) ( $12 \%$ versus $3.3 \%, p=0.005$ for mothers). Smoker fathers were significantly higher among cases than controls (68.7\% vs. $57 \%,(p=0.025)$.

Table (2) shows that parents' consanguinity was significantly higher among cases than controls (60.7\% versus $43.3 \%, p=0.003)$. Twin pregnancy and pre-eclampsia were significantly higher among cases than controls ( $p=0.004$ and 0.003 respectively). Normal delivery was more common among controls than cases $(64.7 \%$ versus $52 \%, p<0.001)$. Neonatal problems after delivery were more common among cases than controls $(49.3 \%$ versus $17.3 \%)$. Breastfeeding was significantly higher among controls than cases $(96.7 \%$ versus $78.7 \%$, $p<0.001)$. Admission into incubator was significantly higher among cases than controls $(36.7 \%$ versus $19.3 \%,(p=0.001)$. Mean number of family members was significantly higher among cases than controls (4.3 versus 4.1) $(p=0.025)$.

Table (3) shows that past history of chicken pox, convulsions and hospital admission was significantly higher among cases than controls ( $p=$ $0.032,0.018$ and 0.001 respectively). Family history of delayed speech among brothers, sisters or other family members was significantly higher among cases than controls ( $p=0.001$ and $<0.001$ respectively).

Table (4) shows logistic regression analysis for variables related to delayed speech. Urban residence, low birth weight, male gender, delivery by Cesarean section, parents' consanguinity and presence cyanosis after birth were significantly associated with delayed speech development.
Table (1): Socio-demographic characteristics of the studied children, Assiut University Hospitals, 2016.

\begin{tabular}{|c|c|c|c|c|}
\hline Characteristics & $\begin{array}{c}\text { Cases } \\
(\mathrm{n}=150)\end{array}$ & $\begin{array}{l}\text { Controls } \\
(n=150)\end{array}$ & $\begin{array}{c}p- \\
\text { value }\end{array}$ & $\begin{array}{c}\text { Odds ratio } \\
(95 \% \mathrm{CI})\end{array}$ \\
\hline \multicolumn{5}{|l|}{ - Gender: } \\
\hline - Male & $105(70.0)$ & $77(51.3)$ & 0.001 & 2.21 \\
\hline - Female & $45 \quad(30.0)$ & $73(49.7)$ & & $(1.38-3.55)$ \\
\hline \multicolumn{5}{|l|}{ •Age: } \\
\hline - $($ Mean $\pm \mathrm{SD})$ & $49.2 \pm 5.8$ & $48.9 \pm 10.2$ & $1.00 *$ & - \\
\hline - Range & $36-60$ & $36-60$ & & \\
\hline \multicolumn{5}{|l|}{ - Residence: } \\
\hline - Urban & $40 \quad(26.7)$ & $12 \quad(8.0)$ & $<0.001$ & 4.18 \\
\hline - Rural & $110(73.3)$ & $138(92.0)$ & & $(2.09-8.35)$ \\
\hline \multicolumn{5}{|l|}{ - Family type: } \\
\hline - Nuclear & $122(81.3)$ & $126(84.0)$ & 0.542 & - \\
\hline - Extended & $28 \quad(18.7)$ & $24 \quad(16.0)$ & & \\
\hline \multicolumn{5}{|l|}{ - Birth order: } \\
\hline - 1st & $92(61.3)$ & $58(38.7)$ & $<0.001$ & - \\
\hline - 2nd-3rd & $34(22.7)$ & $48(32.0)$ & & \\
\hline-4 th-5th & $15(10.0)$ & $18(12.0)$ & & \\
\hline - 6th and more & $9 \quad(6.0)$ & $26(17.3)$ & & \\
\hline \multicolumn{4}{|l|}{ - Father's } & education: \\
\hline $\begin{array}{l}\text { - Illiterate/read } \\
\text { \& write }\end{array}$ & $15(10.0)$ & $6 \quad(4.0)$ & 0.088 & - \\
\hline $\begin{array}{l}\text { - Basic } \\
\text { education }\end{array}$ & $22(14.7)$ & $33(22.0)$ & & \\
\hline - Secondary & $28(18.7)$ & $32(21.3)$ & & \\
\hline $\begin{array}{l}\text { - University or } \\
\text { higher }\end{array}$ & $85(56.7)$ & $79(52.7)$ & & \\
\hline \multicolumn{4}{|l|}{-Mother's } & education: \\
\hline $\begin{array}{l}\text { - Illiterate/Read } \\
\text { \& write }\end{array}$ & $21(14.0)$ & $14(9.3)$ & 0.001 & - \\
\hline $\begin{array}{l}\text { - Basic } \\
\text { education }\end{array}$ & $52(34.7)$ & $84(56.0)$ & & \\
\hline - Secondary & $37(24.7)$ & $32(21.3)$ & & \\
\hline $\begin{array}{l}\text { - University or } \\
\text { higher }\end{array}$ & $40(26.7)$ & $20(13.3)$ & & \\
\hline \multicolumn{5}{|l|}{ - Father' } \\
\hline - Employee & $86(57.3)$ & $71(47.3)$ & 0.021 & - \\
\hline - Farmer & $12(8.0)$ & $18(12.0)$ & & \\
\hline - Skilled worker & $14(9.3)$ & $23(15.3)$ & & \\
\hline $\begin{array}{l}\text { - Unskilled } \\
\text { worker }\end{array}$ & $38(25.3)$ & $38(25.3)$ & & \\
\hline \multicolumn{5}{|l|}{-Mother's } \\
\hline $\begin{array}{l}\text { occupation: } \\
\text { - Employee }\end{array}$ & $18 \quad(12.0)$ & $5 \quad(3.3)$ & 0.005 & 0.25 \\
\hline - Housewife & $132(88.0)$ & $145(96.7)$ & & $(0.09-0.70)$ \\
\hline $\begin{array}{l}\text { - Father's } \\
\text { smoking (yes) }\end{array}$ & $103(68.7)$ & $84(56.0)$ & 0.024 & $\begin{array}{c}2.2 \\
(1.13-4.5)\end{array}$ \\
\hline \multicolumn{5}{|l|}{$\begin{array}{l}\text { - Socio-economic } \\
\quad \text { level: }\end{array}$} \\
\hline - Low & $26(17.3)$ & $26 \quad(17.3)$ & 0.182 & - \\
\hline - Middle & 97 (64.7) & $108(72.0)$ & & \\
\hline - High & $27(18.0)$ & 16 (10.7) & & \\
\hline
\end{tabular}


Table (2): Pre-natal, natal and postnatal risk factors among studied children, Assiut University Hospitals, 2016.

\begin{tabular}{|c|c|c|c|c|}
\hline Characteristics & $\begin{array}{c}\text { Cases } \\
(\mathrm{n}=150)\end{array}$ & $\begin{array}{c}\text { Controls } \\
(\mathrm{n}=150)\end{array}$ & $\begin{array}{c}p- \\
\text { value }\end{array}$ & $\begin{array}{l}\text { Odds ratio } \\
(95 \% \mathrm{CI})\end{array}$ \\
\hline $\begin{array}{l}\text { - Parents' } \\
\text { consanguinity } \\
\text { (yes). }\end{array}$ & $\begin{array}{l}91 \\
(60.7)\end{array}$ & $\begin{array}{l}65 \\
(43.3)\end{array}$ & 0.003 & $\begin{array}{c}2.02 \\
(1.27-3.19)\end{array}$ \\
\hline \multicolumn{5}{|c|}{$\begin{array}{l}\text { - Maternal age at } \\
\text { birth: } \\
\text { - (Mean } \pm \text { SD). } \quad 26.1 \pm 6.6\end{array}$} \\
\hline $\begin{array}{l}\text { - Twin/multiple } \\
\text { pregnancy. }\end{array}$ & $13(8.7)$ & $1(0.7)$ & 0.004 & $\begin{array}{c}3.72 \\
(1.70-9.81)\end{array}$ \\
\hline $\begin{array}{l}\text { - Pre-eclampsia } \\
\text { (yes). }\end{array}$ & $17(11.3)$ & $4(2.7)$ & 0.003 & $\begin{array}{c}4.66 \\
(1.53-4.21)\end{array}$ \\
\hline $\begin{array}{l}\text { - Preterm delivery } \\
\text { (yes). }\end{array}$ & $3(2.0)$ & $1(0.7)$ & 0.315 & - \\
\hline \multicolumn{5}{|l|}{ - Delivery mode: } \\
\hline $\begin{array}{l}\text { - Normal vaginal } \\
\text { delivery. } \\
\text { - Elective } \\
\text { caesarian } \\
\text { section. }\end{array}$ & $\begin{array}{l}78 \\
(52.0) \\
39 \\
(26.0)\end{array}$ & $\begin{array}{l}97 \\
(64.7) \\
10 \\
(6.7)\end{array}$ & $<0.001$ & $\begin{array}{c}0.592 \\
(0.372-0.941)\end{array}$ \\
\hline $\begin{array}{l}\text { - Mandatory } \\
\text { caesarian } \\
\text { section. }\end{array}$ & $\begin{array}{l}33 \\
(22.0)\end{array}$ & $\begin{array}{l}43 \\
(28.7)\end{array}$ & & \\
\hline \multicolumn{5}{|l|}{ - Birth } \\
\hline $\begin{array}{l}\text { - Physician. } \\
\text { - Nurse/midwife. }\end{array}$ & $\begin{array}{l}128(85.3) \\
22(14.7)\end{array}$ & $\begin{array}{l}115(76.7) \\
35 \quad(23.3)\end{array}$ & 0.056 & - \\
\hline \multicolumn{5}{|l|}{$\begin{array}{c}\text { - Place of } \\
\text { delivery: }\end{array}$} \\
\hline $\begin{array}{l}\text { - Hospital. } \\
\text { - Private clinic. } \\
\text { - Home. }\end{array}$ & $\begin{array}{l}76(50.7) \\
52(34.7) \\
22(14.7)\end{array}$ & $\begin{array}{l}75(50.0) \\
40(26.7) \\
35(23.3)\end{array}$ & 0.103 & - \\
\hline $\begin{array}{l}\text { - Problems during } \\
\text { delivery. }\end{array}$ & $9(6.0)$ & $8(5.3)$ & 0.803 & - \\
\hline $\begin{array}{l}\text { - Neonatal } \\
\text { problems after } \\
\text { delivery. }\end{array}$ & $\begin{array}{l}74 \\
(49.3)\end{array}$ & $\begin{array}{l}26 \\
(17.3)\end{array}$ & $<0.001$ & $\begin{array}{c}14.14 \\
(1.83-9.51)\end{array}$ \\
\hline \multicolumn{5}{|l|}{$\begin{array}{l}\text { - Neonatal } \\
\text { problems after } \\
\text { delivery*: }\end{array}$} \\
\hline $\begin{array}{l}\text { - Did not cry } \\
\text { immediately. }\end{array}$ & $13(8.7)$ & $1(0.7)$ & 0.001 & $\begin{array}{c}14.14 \\
(1.83-9.51)\end{array}$ \\
\hline - Cyanosis. & $12(8.0)$ & $1 \quad(0.7)$ & 0.002 & $\begin{array}{c}12.95 \\
(1.66-00.95)\end{array}$ \\
\hline $\begin{array}{l}\text { - Low birth } \\
\text { weight. }\end{array}$ & $73(48.7)$ & $23(15.3)$ & $<0.001$ & $\begin{array}{c}5.24 \\
(3.03-9.05)\end{array}$ \\
\hline $\begin{array}{l}\text { - Difficulty } \\
\text { breathing. }\end{array}$ & $8 \quad(5.3)$ & $4 \quad(2.7)$ & 0.239 & \\
\hline - Breastfeeding. & $118(78.7)$ & $145(96.7)$ & $<0.001$ & $\begin{array}{c}2.53 \\
(1.64-4.12)\end{array}$ \\
\hline $\begin{array}{l}\text { - Admission into } \\
\text { incubator. }\end{array}$ & $55(36.7)$ & $29(19.3)$ & 0.001 & - \\
\hline \multicolumn{5}{|l|}{$\begin{array}{l}\text { - Neonatal } \\
\text { problems } * \text { : }\end{array}$} \\
\hline $\begin{array}{l}\text { - Recurrent } \\
\text { vomiting. }\end{array}$ & $61 \quad(40.7)$ & $31 \quad(21.7)$ & $<0.001$ & $\begin{array}{c}2.63 \\
(1.58-4.39)\end{array}$ \\
\hline - Non feeding. & $28 \quad(18.7)$ & $9(6.0)$ & 0.001 & $\begin{array}{c}3.60 \\
1.63-7.92)\end{array}$ \\
\hline $\begin{array}{l}\text { - No. of family } \\
\text { members (Mean } \\
\pm \text { SD). }\end{array}$ & $4.3 \pm 1.5$ & $4.1 \pm 1.4$ & $0.025^{* *}$ & - \\
\hline $\begin{array}{l}\text { - Child lives with } \\
\text { single parent. }\end{array}$ & $21(14.0)$ & $15(10.0)$ & 0.286 & $\begin{array}{c}0.68 \\
(0.34-1.38)\end{array}$ \\
\hline
\end{tabular}

*: More one cause or problem may be present.

Chi square test was used.

**: $t$-test was used.
Table (3): History of medical conditions among studied children, Assiut University Hospitals, 2016.

\begin{tabular}{|c|c|c|c|c|}
\hline Past/family history & $\begin{array}{c}\text { Cases } \\
(\mathrm{n}=150)\end{array}$ & $\begin{array}{l}\text { Controls } \\
(n=150)\end{array}$ & $\begin{array}{c}p- \\
\text { value }\end{array}$ & $\begin{array}{l}\text { Odds ratio } \\
(95 \% \mathrm{CI})\end{array}$ \\
\hline - Chickenpox. & $7(4.7)$ & $1(0.7)$ & 0.032 & $\begin{array}{c}7.294 \\
(0.886-60.029)\end{array}$ \\
\hline $\begin{array}{l}\text { - Frequent throat } \\
\text { inflammation. }\end{array}$ & $140(93.3)$ & $144(96.0)$ & 0.304 & \\
\hline • Pneumonia. & $20(13.3)$ & $14(9.3)$ & 0.274 & - \\
\hline - Meningitis. & $4 \quad(2.7)$ & $2(1.3)$ & 0.409 & - \\
\hline - Bronchial asthma. & $57(38.0)$ & $51(34.0)$ & 0.470 & \\
\hline • Convulsions. & $10(6.7)$ & $2(1.3)$ & 0.018 & $\begin{array}{c}5.28 \\
(1.14-24.55)\end{array}$ \\
\hline - Head trauma. & $1 \quad(0.7)$ & $2(1.3)$ & 0.652 & - \\
\hline • Hospital admission. & $21(14.0)$ & $5(3.3)$ & 0.001 & $\begin{array}{c}4.72 \\
(1.73-12.88)\end{array}$ \\
\hline $\begin{array}{l}\text { - Brothers or sisters } \\
\text { had DLD. }\end{array}$ & $10(6.7)$ & $0(0.0)$ & 0.001 & $\begin{array}{c}2.071 \\
(1.839-2.334)\end{array}$ \\
\hline $\begin{array}{l}\text { - Other family } \\
\text { member had DLD }\end{array}$ & $51(34.0)$ & $3(2.0)$ & $<0.001$ & $\begin{array}{c}25.24 \\
(7.66-83.13)\end{array}$ \\
\hline $\begin{array}{l}\text { - Mother had mental } \\
\text { or psychological } \\
\text { disorder. }\end{array}$ & $\begin{array}{l}1(0.7) \\
7\end{array}$ & $3(2.0)$ & 0.314 & \\
\hline $\begin{array}{l}\text { - Father had mental or } \\
\text { psychological } \\
\text { disorder. }\end{array}$ & $(4.7)$ & $3(2.0)$ & 0.198 & - \\
\hline $\begin{array}{l}\text { - Sitting for long time } \\
\text { to watch TV (yes). }\end{array}$ & $43(28.7)$ & $91(60.7)$ & $<0.001$ & $\begin{array}{c}0.26 \\
(0.16-0.42)\end{array}$ \\
\hline
\end{tabular}

Chi square test was used.

Table (4): Logistic regression analysis for variables related to delayed speech among studied children, Assiut University Hospitals, 2016.

\begin{tabular}{llc}
\hline Variables & \multicolumn{1}{c}{ OR $(95 \%$ CI $)$} & $p$-value \\
\hline - Residence (urban). & $8.549(3.338-21.896)$ & 0.000 \\
- Low birth weight. & $6.793(3.408-13.540)$ & 0.000 \\
- Gender (males). & $4.219(1.355-12.956)$ & 0.026 \\
- Delivery mode (normal): & $2.600(1.238-5.460)$ & 0.000 \\
- Elective C.S. & $7.931(2.939-21.404)$ & 0.012 \\
- Mandatory C.S. & $3.621(1.896-6.914)$ & 0.000 \\
- Parent consanguinity. & & 0.000 \\
- Birth order & $3.984(1.480-10.724)$ & 0.006 \\
- 1st & $1.675(0.603-4.657)$ & 0.323 \\
- 2nd-3rd & $1.990(0.609-6.499)$ & 0.254 \\
- 4th-5th & $2.754(1.292-5.870)$ & 0.009 \\
- Cyanosis & 0.026 & 0.000 \\
- Constant & & \\
\hline
\end{tabular}

R square: 44.3 .

\section{Discussion}

The current study documented that dominance of speech delay in boys than girls. This gender discrepancy was found consistently in many studies [11-17]. Geschwind et al., (1985) revealed that boys are more vulnerable to delayed language development and explained this by slow maturation of the nervous system among boys and the negative impact of testosterone on the development of areas essential for the speech skills [18]. 
Moreover, this study revealed that urban participants were risky for delayed language development. This result was consistent with Mishra, 2015 [19]. This finding might be due to unsocial lifestyle in urban area compared to rural. Also, nowadays, urban people prefer indoor activities and excessive use of electronic devices which could be another reason. In addition, most of the mothers in urban areas do not have enough time to spend in playing and speaking with their children.

The current study revealed that significant association between low birth weight and delayed speech. Several studies documented the same findings $[12,17,20-23]$.

In addition, our finding reported that children who were delivered by cesarean section was significantly at a greater risk to have delayed speech. The same finding was reported by other studies $[24,25]$

This study found that consanguineous marriage was significantly associated with DLD. Many studies documented the same result [26-28].

Furthermore, the current study showed that the first child was more vulnerable to DLD. The same was reported by different studies [13,17,29]. Eckstein, 2000 revealed that higher incidence of problematic behaviors and limitations in social skills of first child than others these characteristics may be due to parental over-protection and excessive monitoring [30]

The results of this study revealed that children exposed to cyanosis were significantly at a greater risk than noncyanotic children. Many studies reported the same findings $[\mathbf{2 2 2 , 2 3 , 3 1 , 3 2 ]}$. This finding may be due to neonates who were exposed to asphyxia neonatorum are more vulnerable to DLD due to injury or malfunctioning of the brain [32]

On the otherhand, socioeconomic level was insignificantly associated with DLD. This finding was contestant with other studies $[33,34]$ but other studies reported significant association $[\mathbf{1 5 , 3 5}]$. This discrepancy could be due to the different place, socio demographic characteristics of participants or different scale of classification.

The results of this study showed that otitis media did not significantly increase the risk of DLD. This finding was consistent with other studies $[35,36]$. The effect of otitis media may depend on its frequency and severity and proper treatment.

Additionally, the result of this study revealed that family history of DLD was important risk factor (bivariate analysis), the same finding was recorded by many studies $[\mathbf{8 , 1 4 , 1 6 , 3 3 ]}$. This finding may be due to genetic factor or because of family member exposed to the same environmental influences or to a combination of both.

The result of this study showed that preterm delivery was risk factor of DLD especially in the perinatal period (bivariate analysis), the same was recorded by other studies $[\mathbf{1 4 , 2 2 , 3 6 ]}$. Which state that the delay in physiological and neurobiological maturation is accompanied by delay in language development. On the other hand, Nivedita et al., [33] recorded insignificant association of preterm and DLD.

\section{Conclusion and Recommendations:}

It was concluded that the significant risk factors of DLD are urban residence, low birth weight, male gender, delivery by Cesarean section, parent consanguinity and presence of cyanosis after birth.

It was recommended to raise the awareness about risk factors of DLD among parents, primary health care providers, pediatricians and phoniatric specialists for prevention, early detection and proper management.

\section{Acknowledgement:}

The authors would like to acknowledge the support of all staff members of outpatient clinic of phoniatric unit for helping in accomplishment of this research work especially in diagnosis the cases of DLD.

\section{References}

1- LAUGHLIN M.C. MAURAR: Speech and language delay in children. Am. Fam. Physician, 15; 83 (10): 1183-8, 2011.

2- LAW J., BOYLE J., HARRIS F., HARKNESS A. and NYE C.: Prevalence and natural history of primary speech and language delay: Findings from a systematic review of the literature. Int. J. Lang. Commun. Disord., 35 (2): $165-88,2000$.

3- RUBEN R.J.: Redefining the survival of the fittest: Communication disorders in the 21 st century. The Laryngoscope, 110; 241-5, 2000.

4- ALBERT L. SIU: Screening for Speech and Language Delay and Disorders in Children Aged 5 Years or Younger: US Preventive Services Task Force Recommendation Statement. Pediatrics, 136 (2): e474-81. http://pediatrics. aappublications.org/content/136/2/e474 last accessed on 4/1/2018, 2015.

5- BASHIR A.S. and SCAVUZZO A.: Children with language disorders: Natural history and academic success. J. Learn. Disabil., 25 (1): 53-65, discussion 66-70, 1992. 
6- RAMEY C. and CAMPBELL F.A.: Preventative education for high risk children: Cognitive consequences of the Carolina Abecedarian Project. American Journal of Mental Deficiency, 88: 515-23, 1984.

7- TOMBLIN J.B., HARDY J. and HEIN H.: Predicting poor communication status in preschool children using risk factors present at birth. Journal of Speech and Hearing Research, 34: 1096-105, 1991.

8- CHAIMAY B., THINKHAMROP B. and THINKHAMROP J.: Risk factors associated with Language development problems in childhood-a literature review. J. Med. Assoc. Thai., 89: 1080-6, 2006.

9- SCHIRMER C.R., PORTUGUEZ M.W. and NUNES M.L.: Clinical assessment of language development in children at age 3 years that were born preterm. Arq. Neuro. Psiquiatr., 64 (4): 926-31, 2006.

10- EL-GILANY A., EL-WEHADY A. and EL-WASIFY M.: Updating and validation of the socioeconomic status scale for health research in Egypt, Eastern_Mediterranean_ Health_Journal, 18 (9): 962-8, 2012.

11- CAMPBELL T.F., DOLLAGHAN C.A., ROCKETTE H.E., PARADISE J.L., FELDMAN H.M., SHRIBERG L.D., SABO D.L. and KURS-LASKY M.: Risk factors for speech delay of unknown origin in 3-year-old children. child development, 74 (2): 346-57, 2013.

12- MONDAL N., BHAT B.V., PLAKKAL N., THULASINGAM M., AJAYAN P. and POORNA D.R.: Prevalence and risk factors of speech and language delay in children less than 3 years of age. J. Compr. Ped., 7 (2): e33173, 2016.

13- SILVA G.M.D., COUTO M.I.V. and MOLINIAVEJONAS D.R.: Risk factors identification in children with speech disorders: Pilot study. CoDAS, 25 (5): 45662, 2013.

14- REILLY S., WAKE M., UKOUMUNNE O.C., BAVIN E., PRIOR M., CINI E., et al.: Predicting language outcomes at 4 years of age: Findings from early language in Victoria study. Pediatrics, 126 (6): e1530-7, 2010.

15-CHO J., HOLDITCH-DAVIS D. and MILES M.S.: Effects of gender on the health and development of medically atrisk infants. J. Obstet. Gynecol. Neonatal. Nurs., 39 (5): 536-49, 2010

16- CONTI-RAMSDEN G. and DURKIN K.: What Factors Influence Language Impairment? Considering Resilience as well as Risk. Folia Phoniatr. Logop., 67: 293-9, 2015.

17- MOLINI-AVEJONAS D.R., FERREIRA L.V. and De La HIGUERA AMATO C.A.: Risk Factors for SpeechLanguage Pathologies in Children. DOI:10.5772/ intechopen.70107, 2017.

18- GESCHWIND N. and GALABURDA A.M.: Cerebral lateralization. Biological mechanisms, associations, and pathology: I. A hypothesis and a program for research. Arch. Neurol., 42 (5): 428-59, 1985.

19- MISHRA A.: Assessment of speech and language delay among 0-3 year old children attending well baby clinics using Language Evaluation Scale Trivandrum (LEST 03). European Journal of Paediatric Neurology. Page S36. https://doi.org/10.1016/S1090-3798 (15) 30115-X, 2015.
20- CHRISTINE D.E.F., SARA V.J. and KEITH S.G.: Early Risk Factors for Speech and Language Impairment. Lawrence Erlbaum Association Inc., 13 (3): 173-91, 2015.

21- ARAM D.M., HACK M., HAWKINS S., WEISSMAN B.M. and BORAWSKI-CLARK E.: Very-low-birthweight children and speech and language development. J. Speech Hear. Res., 34: 1169-79, 1991.

22- STANTON-CHAPMAN T.L., CHAPMAN D.A., BAINBRIDGE N.L. and SCOTT K.G.: Identification of early risk factors for language impairment. Research in developmental disabilities, 23 (6): 390-405. Doi:10.1016/s08914222(02)00141-5, 2002.

23- SIDHU M., MALHI P. and JERATH J.: Multiple risks and early language development. The Indian Journal of Pediatrics, 77 (4): 391-5. Doi: 10.1007/s12098-0100044y, 2010.

24- MATTHEW ABE B.Sc. and BPHARM: Incidence of hearing and speech impairment in children born by caesarian section. Pharma news, 2016.

25- AL-FADHLI YAHYA KHADIJA and AL-BUNAIAN A. RAHMAN NADA: Prevalence and Social Influences of Delayed Language Development in Preschool-Age Saudi Children. I.J.S.R.: Volume 6 Issue 8, 2017.

26- MAHESWARI K. and WADHWA LALITA: Role of consanguinity in paediatric neurological disorders. Int. J. Contemp. Pediatr., 3 (3): 939-42, 2016.

27- EL-MELIEGY H.K.E. and EL-SABBAGH M.H.: Etiology of Developmental Delay in Egyptian Children. The International Journal of Child Neuro-Psychiatry Vol. 1 (1), 2014.

28- TAREK J.S., KAMAL D.J. and SERAJ Z.M.: Speech Abnormality among Saudi Arabian Children with Hearing Impairment. Bahrain Medical Bulletin, Vol. 23, No. 1, 2001.

29- CHAIMAY B., THINKHAMROP B. and THINKHAMROP J.: Risk factors associated with language development problems in childhood-a literature review. J. Med. Assoc. Thai., 2; 89 (7): 1080-6, 2006.

30- ECKSTEIN D.: Empirical studies indicating significant birth-order-related personality differences. J. Indiv. Psychol., 56: 481-94, 2000.

31- HINCKELDEY K.W., MESSMER B.J., SEGHAYE M.C. and HUBER W.: Long-term outcome of speech and language in children after corrective surgery for cyanotic or acyanotic cardiac defects in infancy. European Journal of Paediatric Neurology, 12: 378-86, 2008.

32- SHANTHINI S.V.: Language status in children with birth asphyxia: A follow-up study (brain and language). GSTF International Journal of Nursing and Health Care (JNHC), Vol. 1 No. 1, 2013

33- NIVEDITA M., VISHNU B.B., NISHAD, THULASINGAM P.M., PAYYADAKKATH A. and RACHEL P.D.: Prevalence and Risk Factors of Speech and Language Delay in ChildrenLess Than Three Years of Age. J. Compr. Ped., May 7 (2): e33173, 2016.

34- PHILIP W., McFIONA Q., LUCY T. and ALEX C.: Language Delay Is Not Predictable from Available Risk Factors. The Scientific World Journal Vol. 2013 Article ID 947018, 8.http:/dx.doi.org/10.1155/2013/947018, 2013. 
35- CAMPBELL T.F., DOLLAGHAN C.A., ROCKETTE H.E., PARADISE J.L., FELDMAN H.M., et al.: Risk Factors for Speech Delay of Unknown Origin in 3-YearOld Children. Child Development, (74) 2: 346-57, 2003.
36- DUARTE SILVA G.M., COUTO M.V. and MOLINIAVEJONAS D.R.: Risk factors identification in children with speech disorders: Pilot study.CoDAS; 25 (5): 456 62, 2013.

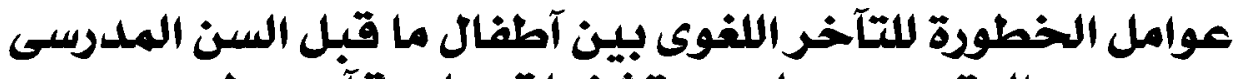

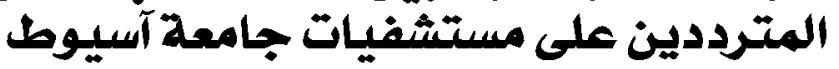

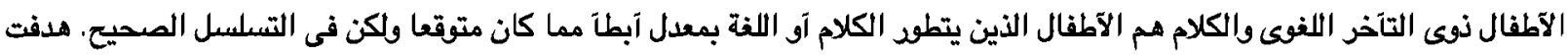

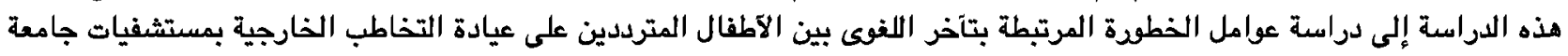

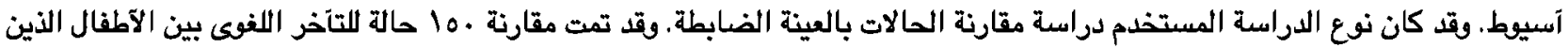

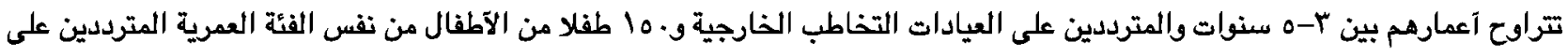

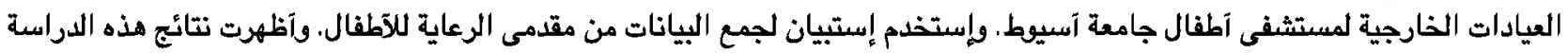

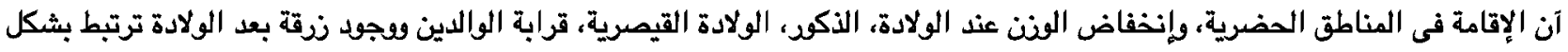

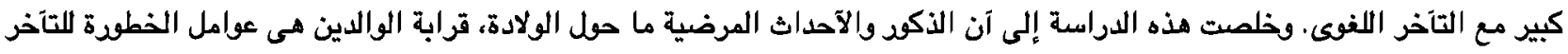
اللغوى. ولهذا يجب رفع الوعى حل عوامل الخطر بالتآخر اللغوى بين الآباءومقدمى الرعاية الصحية للوقاية والكثف المبكر والعلاج السليم. 